\title{
Shape Control of Piezoelectric Bimorph Cantilever Beam Using Comsol Multiphysics and Genetic Algorithm
}

\author{
Ranaivoarison Z. N. \\ Student \\ University of Antananarivo in STII \\ Antananarivo, Madagascar
}

\author{
Andrianaharison Y. \\ Full Professor \\ University of Antananarivo in ESPA \\ Antananarivo, Madagascar
}

\begin{abstract}
This paper presents a numerical study on optimal voltage, optimal load and optimal thickness for static shape control of piezoelectric bimorph cantilever beam. A finite element model using Comsol Multiphysics is developed to characterize the behavior of structure. Genetic Algorithm is used to calculate optimum values.
\end{abstract}

Keywords-Finite Element; Shape control; Piezoelectric; Comsol Multiphysics; Genetic Algorithm.

\section{INTRODUCTION}

Since a few years, investigations on smart structures having integrated self-monitoring and control capabilities were carried out by many researchers. Among the various smart materials, particular attention is given to piezoelectric. Due to the direct and converse effects of piezoelectric materials, they have found applications in sensor and actuator technologies. One of the most important piezoelectric materials is Lead Zirconate Titanate (PZT). H.S. Kim, J.H. Kim, J. Kim [1] used PZT for vibration energy harvesting. Another application of PZT is shape control. The objective is to optimize some parameters in order to achieve the desired shapes. E.P. Hadjigeorgiou, G.E. Stavroulakis, C.V. Massalas [2] worked on shape control of beam using piezoelectric actuator. Genetic optimization is used to control the voltage applied to the actuator. In the paper of H.R. Patel, J.R. Mevada [3], size, location and control voltage of actuators are taken as variables. Optimum values of all variables are calculated with Genetic Algorithm. Initial condition of beam is taken as horizontal position and three higher order polynomial curves are taken as desired shapes for beam to achieve. Piezoelectric bimorph cantilever beam was studied by L.N. Sulbhewar, P. Raveendranath [4]. A Timoshenko piezoelectric beam finite element based on Firstorder Shear Deformation Theory (FSDT) was developed. It is an improved model of the FSDT proposed by S. Narayanan, V. Balamurugan [5].

In the present work, piezoelectric bimorph cantilever beam is investigated using Comsol Multiphysics and the results are compared to analytical results based on FSDT. Genetic Algorithm carried out using Matlab works with Comsol Multiphysics to optimize voltage, load and thickness for shape control. Error between desired shape and achieved shape is taken as an objective function.

\section{METHODOLOGY}

We firstly introduce the equations which were used to develop the piezoelectric model. This model is validated by comparing the results got using Comsol Multiphysics with analytical results. In a second part, we take advantage of the power of Comsol Multiphysics combined with Matlab [6]. The developed piezoelectric model is used with Genetic Algorithm for shape control.

\section{A. Piezoelectric equations and model in Comsol}

Using the first law of thermodynamic, the internal energy of an electromechanical system can be expressed as:

$$
d U=T_{i j} d S_{i j}+E_{i} d D_{i}
$$

$$
\alpha+\beta=\chi \text {. }
$$

Where:

$U$ : internal energy;

$T_{i j}$ : stress;

$S_{i j}$ : strain;

$E_{i}$ : electric field;

$D_{i}$ : electric displacement.

Electric enthalpy is defining as:

$$
H=U-E_{i} D_{i}
$$

\section{$H$ : Electric enthalpy}

The total differential of $H$ gives us:

$$
d H=T_{i j} d S_{i j}-D_{i} d E_{i}
$$

Equation (3) can be written as:

$$
\begin{gathered}
T_{i j}=\frac{\partial H}{\partial S_{i j}} \\
D_{i}=-\frac{\partial H}{\partial E_{i}}
\end{gathered}
$$

In linear piezoelectricity theory, the form taken by $H$ is: 


$$
H=\frac{1}{2} c^{E_{i j k l}} S_{i j} S_{k l}-e_{k i j} E_{k} S_{i j}-\frac{1}{2} \varepsilon_{i j} E_{i} E_{j}
$$

Where:

$c^{\mathrm{E}}{ }_{i j k l}$ : constant elastic;

$e_{k i j}$ : constant piezoelectric;

$\varepsilon_{i j}^{S}$ : constant dielectric.

The piezoelectric constitutive equations are:

$$
\begin{gathered}
T_{i j}=c^{E_{i j k l}} S_{k l}-e_{k i j} E_{k} \\
D_{i}=e_{i k l} S_{k l}+\varepsilon^{S} E_{i j} E_{j}
\end{gathered}
$$

The piezoelectric model is implemented using Comsol Multiphysics. We consider here a bimorph cantilever beam with oppositely poled piezoelectric layers. The structure has clamped end at the left-hand side. We used three types of material while the geometry is fixed: PZT 4, PZT 5A and PZT $5 \mathrm{H}$. Those materials are available in the library of Comsol Multiphysics.

In order to compare Comsol-based simulation results with analytical results based on FSDT, we investigated static analysis-actuator configuration and static analysis-sensor configuration.

- Static analysis-actuator configuration:

Interface of the bimorph is grounded and the voltages of $\pm 10 \mathrm{~V}$ are applied on the free surfaces.

- Static analysis-sensor configuration:

The piezoelectric bimorph beam is subjected to a tip load of $1000 \mathrm{~N}$.

Displacements of the piezoelectric bimorph cantilever beam are given in the results of Comsol Multiphysics. Tip deflection is compared to analytical results for the three materials.

A schematic view of the piezoelectric bimorph cantilever beam is presented in Fig. 1.

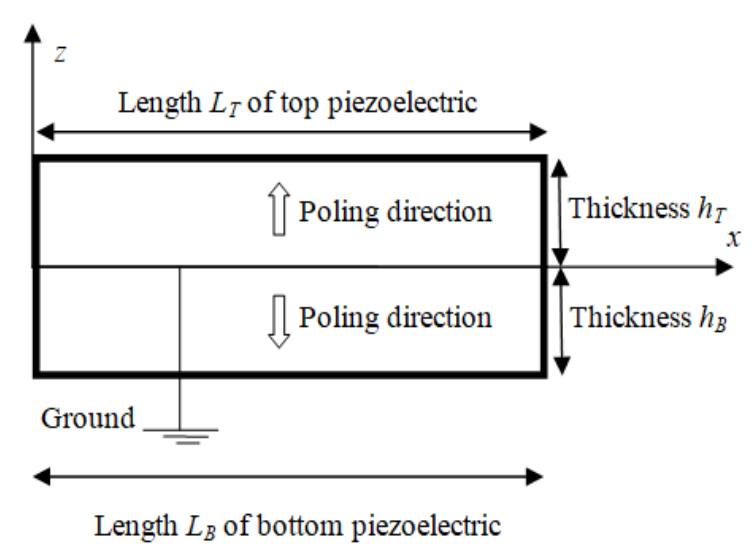

Fig. 1. Piezoelectric bimorph cantilever beam

Dimensions of the top and bottom piezoelectric are the same and given in TABLE I.
TABLE I. DIMENSIONS OF TOP AND BOTTOM PIEZOELECTRIC

\begin{tabular}{|l|c|c|}
\hline & Top piezoelectric & Bottom Piezoelectric \\
\hline Length $\left(\times 10^{-3} \mathrm{~m}\right)$ & $L_{T}=100$ & $L_{B}=100$ \\
\hline Thickness $\left(\times 10^{-3} \mathrm{~m}\right)$ & $h_{T}=5$ & $h_{B}=5$ \\
\hline
\end{tabular}

B. Shape control

The model developed in Comsol Multiphysics is used with Genetic Algorithm for shape control. Initial condition is taken as horizontal. Desired shape is taken as polynomial curve. Error between desired shape and achieved shape is to be considered as an objective function to minimize. Error can be expressed as:

$$
\text { Error }=\sqrt{\sum_{i}\left(\gamma_{i}-q_{i}\right)^{2}}
$$

Where:

$\gamma_{i}$ : pre-defined displacement;

$q_{i}$ : achieved displacement;

Genetic Algorithm is a class of probabilistic algorithm based on biological evolution. It starts working on a randomly generated set of solutions, known as population. The solution is called chromosome. Genetic Algorithm generates new population of chromosomes by selecting the better fit solutions from existing population and applying genetic operators (crossover, mutation) to produce new offspring of the solutions.

Here, Genetic Algorithm is carried out using Matlab.

Methodology associating Comsol Multiphysics and Matlab is summarized in Fig. 2.

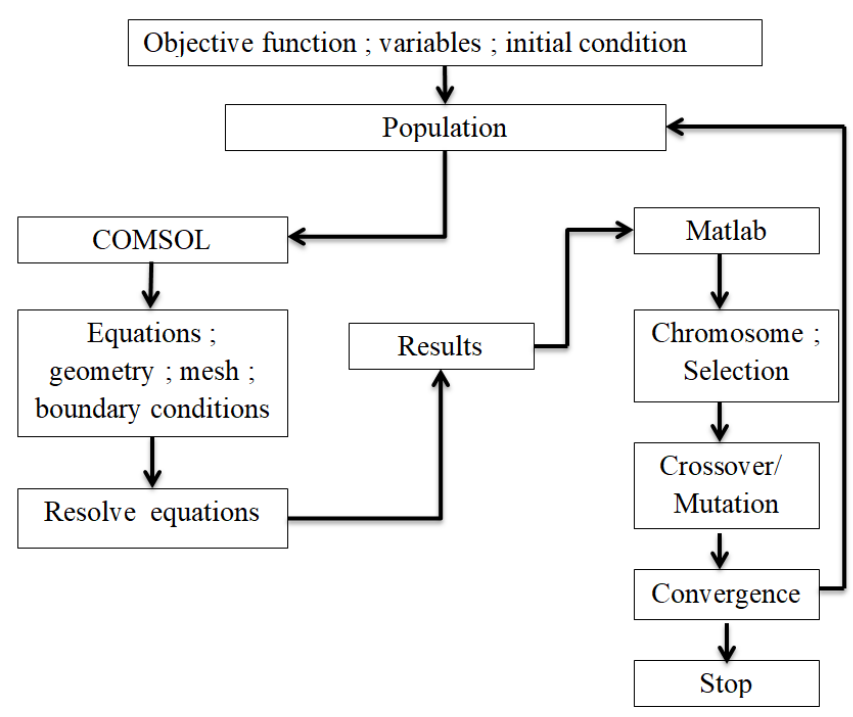

Fig. 2. Methodology using Comsol Multiphysics and Matlab

For Genetic algorithm, population is set as 100 for all cases. Number of generation is allowed as 50 .

In the shape control, PZT 4 is used as piezoelectric material. We consider three kinds of shape control problems with various boundary conditions: the voltage problem, the tip load problem, the voltage and thickness problem. 
- Voltage problem:

The first problem consists in finding voltage $\phi$ which minimizes the objective function under the constraint:

$\phi$ : Actuation voltage

$$
\phi_{\min } \leq \phi \leq \phi_{\max }
$$

$\phi_{\min }:$ Lower saturation voltage

$\phi_{\text {max }}$ : Upper saturation voltage

In order to test the efficiency of methodology associating both Comsol Multiphysics and Matlab, the desired shape that we considered in the voltage problem is displacement corresponding to $\pm 50 \mathrm{~V}$ as shown in Fig. 3 .

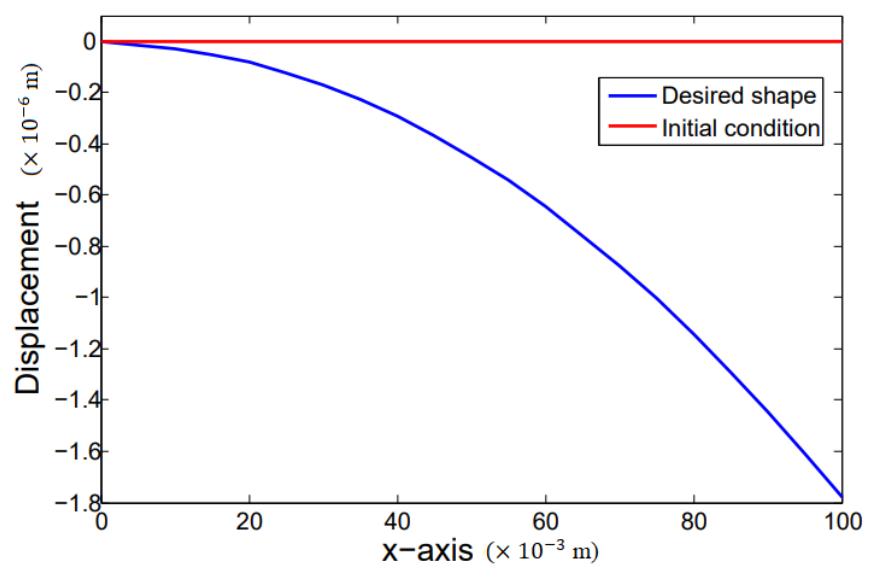

Fig. 3. Initial condition and desired shape

- Tip load problem:

The desired shape used above is the same considered in the tip load problem. It consists to find tip load which minimizes the objective function.

This work shows how tip load are depended on the desired shape.

- Voltage and thickness:

In this problem, two variables are considered: voltage and thickness. The thickness of top piezoelectric $h_{T}$ is varied while the thickness of bottom piezoelectric $h_{B}$ is fixed. We investigated two cases:

Thickness is under constraints: $\left.1 \times 10^{-3} \mathrm{~m}\right) \leq h_{T} \leq 4 \times 10^{-3} \mathrm{~m}$

Thickness is under constraint $\left.6 \times 10^{-3} \mathrm{~m}\right) \leq h_{T} \leq 15 \times 10^{-3} \mathrm{~m}$.

The first case corresponds to thickness of top piezoelectric inferior to the thickness of bottom piezoelectric. The second case corresponds to thickness of top piezoelectric superior to the thickness of bottom piezoelectric.

This work shows how thickness and control voltage are depended on the desired shape.

\section{RESULTS AND DISCUSSIONS}

\section{A. Comparison of tip deflection got in model Comsol and analytical results}

The displacement caused by an applied voltage $\pm 10 \mathrm{~V}$ is shown in Fig. 4 and the displacement caused by a tip load of $1000 \mathrm{~N}$ is shown in Fig. 5 for PZT 4, PZT 5A, PZT 5H.

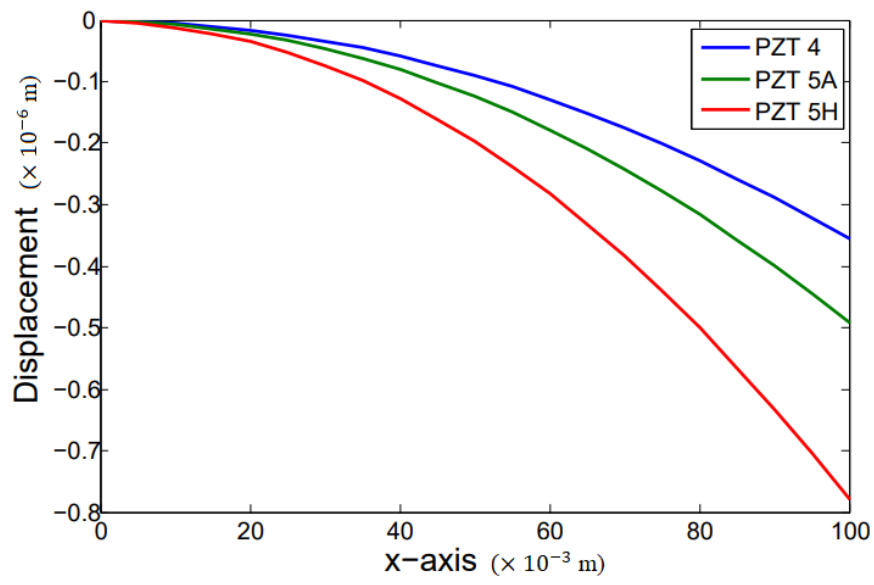

Fig. 4. Displacement by $\pm 10 \mathrm{~V}$

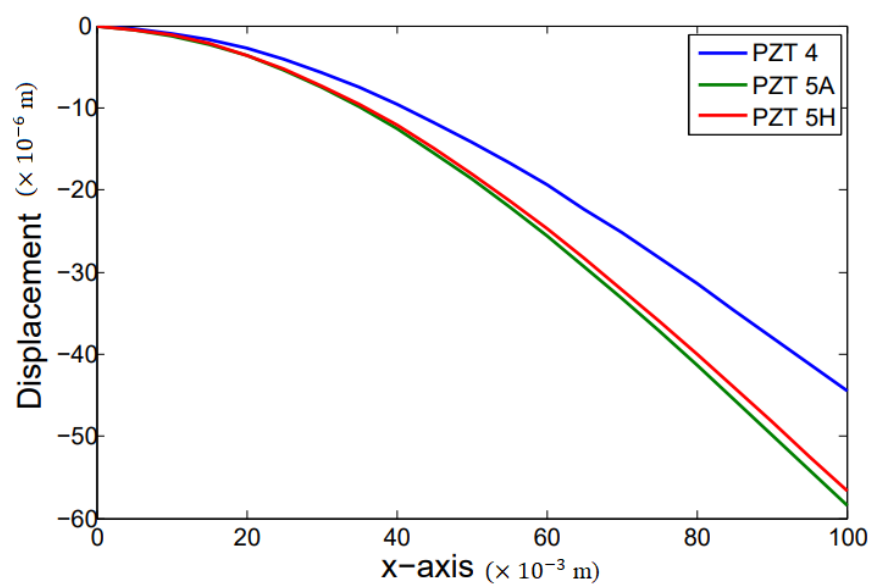

Fig. 5. Displacement by a tip load of $1000 \mathrm{~N}$

Absolute tip deflection of different piezoelectric materials got in Comsol is in agreement with analytical results based on FSDT [4] as shown in the TABLE II and TABLE III.

TABLE II. COMPARISON OF TIP DEFLECTION CAUSED BY AN APPLIED VOLTAGE $\pm 10 \mathrm{~V}$

\begin{tabular}{|c|c|c|c|}
\hline Materials & $\begin{array}{c}\text { FSDT } \\
\left(\times 10^{-6} \mathrm{~m}\right)\end{array}$ & $\begin{array}{c}\text { Comsol } \\
\left(\times 10^{-6} \mathrm{~m}\right)\end{array}$ & $\begin{array}{c}\text { Error percentage } \\
(\%)\end{array}$ \\
\hline PZT 4 & 0.3620 & 0.3559 & 1.68 \\
\hline PZT 5A & 0.5023 & 0.4927 & 1.91 \\
\hline PZT 5H & 0.7899 & 0,7801 & 1.24 \\
\hline
\end{tabular}

TABLE III. COMPARISON OF TIP DEFLECTION CAUSED BY A TIP LOAD OF $1000 \mathrm{~N}$

\begin{tabular}{|c|c|c|c|}
\hline Materials & $\begin{array}{c}\text { FSDT } \\
\left(\times 10^{-6} \mathrm{~m}\right)\end{array}$ & $\begin{array}{c}\text { Comsol } \\
\left(\times 10^{-6} \mathrm{~m}\right)\end{array}$ & $\begin{array}{c}\text { Error percentage } \\
(\%)\end{array}$ \\
\hline PZT 4 & 46.061 & 44.484 & 3.42 \\
\hline PZT 5A & 60.942 & 58.486 & 4.03 \\
\hline PZT 5H & 57.156 & 56.625 & 0.92 \\
\hline
\end{tabular}

B. Genetic Algorithm and Comsol used for shape control

- Voltage problem

This first problem is used to test the efficiency of the methodology associating Comsol Multiphysics and Matlab. The desired shape corresponds to displacement caused by an applied voltage $\pm 50 \mathrm{~V}$.

Constraints: $0 \leq \phi \leq 100$ 
Convergence with error equal to $1.34 * 10^{-4}$ is achieved at $15^{\text {th }}$ iteration.

Error is plotted with number of generations in Fig.6.

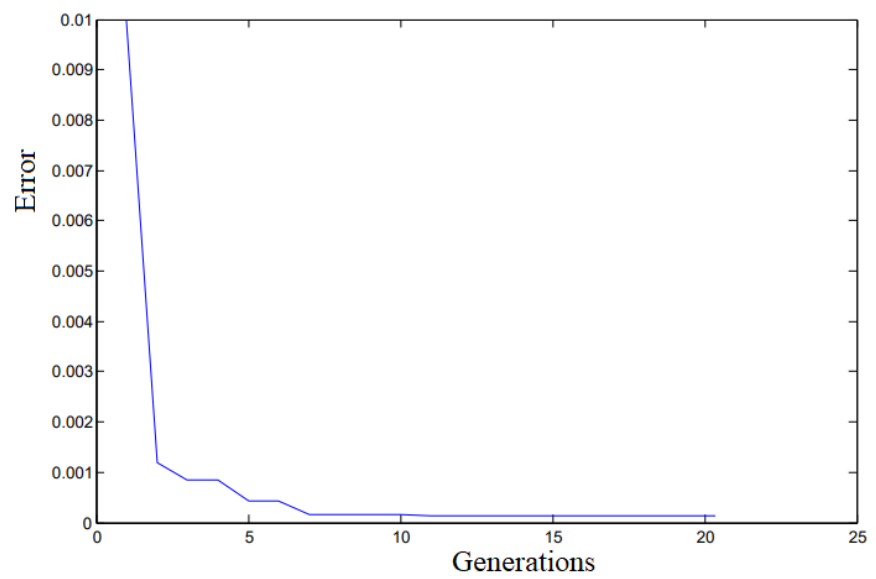

Fig. 6. Error for control voltage

\section{- Tip Load problem}

Here, the same desired shape used previously is considered. Tip load is taken as variable. The tip load which can produce the desired shape is $37 \mathrm{~N}$.

- Voltage and thickness problem

Thickness of bottom piezoelectric is fixed. The Thickness of top piezoelectric $h_{T}$ and voltage $\phi$ are variables.

- Case 1: thickness of top piezoelectric is taken as inferior of the thickness of bottom piezoelectric.

Constraints: $1 \times 10^{-3} \mathrm{~m} \leq h_{T} \leq 4 \times 10^{-3} \mathrm{~m}$ and $0 \leq \phi \leq 100$

Optimum values: $h_{T}=4 \times 10^{-3} \mathrm{~m}$ and $\phi=40 \mathrm{~V}$.

If we compare applied voltage got in this case with results in the control voltage only, we see that decrease in thickness of top piezoelectric needs a smaller applied voltage to achieve the desired shape.

- Case 2: thickness of top piezoelectric is taken as superior of the thickness of bottom piezoelectric.

Constraints: $6 \times 10^{-3} \mathrm{~m} \leq h_{T} \leq 15 \times 10^{-3} \mathrm{~m}$ and $0 \leq \phi \leq 100$

Optimum values: $h_{T}=6 \times 10^{-3} \mathrm{~m}$ and $\phi=60 \mathrm{~V}$

If we compare applied voltage got in this case with results in the control voltage only, we see that increase in thickness of top piezoelectric needs a greater applied voltage to achieve the desired shape.

\section{CONCLUSION}

Here, methodology using Comsol Multiphysics and Genetic Algorithm is developed for shape control. To minimize control cost of piezoelectric bimorph cantilever beam, it is required to optimize voltage, tip load and thickness on the structure. Optimization is carried out for the error minimization between desired and actual shape with the use of genetic algorithm.

- Tip deflection got with model developed in Comsol Multphysics is in agreement with analytical results based on FSDT;

- Efficiency of methodology associating Comsol Multiphysics and Genetic Algorithm was tested by control voltage and the results are satisfactory.

- An equivalent tip load can cause the same desired shape got by applied voltage

- Decrease in thickness of top piezoelectric needs a smaller applied voltage to achieve the desired shape. Increase in thickness of top piezoelectric needs a greater applied voltage to achieve the desired shape.

\section{REFERENCES}

[1] H.S. Kim, J.H. Kim, and J. Kim, "A review of piezoelectric energy harvesting based on vibration," International Journal of Precision Engineering and Manufacturing, vol. 12, pp. 1129-1141, December 2011.

[2] E.P. Hadjigeorgiou, G.E. Stavroulakis, and C.V. Massalas, "Shape control and damage identification of beams using piezoelectric actuation and genetic optimization," International Journal of Engineering Science, vol. 44, pp. 409-421, April 2006.

[3] H.R. Patel and J.R. Mevada, "Shape control and optimization using cantilever beam," International Journal of Engineering Research and Applications, vol. 3, pp. 155-161, June 2013.

[4] L.N. Sulbhewar and P. Raveendranath, "A Timoshenko piezoelectric beam finite element with consistent performance irrespective of geometric and material configurations," Latin American Journal of Solids and Structures, vol. 13, pp. 992-1015, 2016.

[5] S. Narayanan and V. Balamurugan, "Finite element modelling of piezolaminated smart structures for active vibration control with distributed sensors and actuators," Journal Sound and Vibration, vol. 262, pp.529-562, June 2003.

[6] S.Ozana, M. Pies and R. Hajovsky, "Using matlab and comsol multiphysics for optimization of the model of underground thermal processes at old mining dumps," Applied Mechanics and Materials, vol. 548-549, pp. 571-578, April 2014 